\title{
Condições de saúde de idosos portadores de Diabetes mellitus e hipertensão arterial sistêmica
}

\author{
Marília de Oliveira Silva Ferraz ${ }^{1}$, Luciana Araújo dos Reis ${ }^{2}$, Pollyanna Viana Lima ${ }^{3}$
}

\begin{abstract}
Resumo: O presente estudo tem como objetivo investigar as condições de saúde de idosos portadores de Hipertensão Arterial Sistêmica e de Diabetes Mellitus. Metodologia: Trata-se de um estudo do tipo descritivo, com abordagem quantitativa, realizado com 53 idosos cadastrados no programa Hiperdia. Os instrumentos utilizados para a coleta dos dados foram o Mini-Exame do Estado Mental, o questionário sociodemográfico e econômico e o de condições de saúde. Para realização da análise foi utilizado o programa estatístico SPSS ${ }^{\circ}$, versão 20.0. Resultados: $66,0 \%$ dos idosos são hipertensos e $22,6 \%$ são hipertensos e diabéticos; $86,8 \%$ não possuem sequela; $45,3 \%$ tem histórico de hipertensos na família e $28,3 \%$ de diabetes e hipertensão; $71,7 \%$ não foram internados nos últimos cinco anos; 56,6 consome verduras; $88,7 \%$ seguem a dieta recomendada; $81,10 \%$ não são fumantes; $90,6 \%$ não bebem bebida alcoólica e $49,1 \%$ não praticam atividade física. Conclusão: os idosos têm condições de saúde favoráveis.
\end{abstract}

Descritores: Idoso, Hipertensão arterial, Diabetes mellitus, Condições de saúde.

\section{Health conditions of elderly carriers of Diabetes mellitus and systemic arterial hypertension}

\begin{abstract}
The present study aims to investigate the health conditions of elderly patients with Systemic Arterial Hypertension and Diabetes Mellitus. Methodology: This is a descriptive study, with a quantitative approach, carried out with 53 elderly people enrolled in the Hiperdia program. The instruments used to collect the data were the Mental State Mini-Exam, the socio-demographic and economic questionnaire, and the health conditions questionnaire. Statistical analysis was performed using the statistical software SPSS $®$, version 20.0 . Results: $66.0 \%$ of the elderly are hypertensive and $22.6 \%$ are hypertensive and diabetic; $86.8 \%$ have no sequelae; $45.3 \%$ had a history of hypertension in the family and $28.3 \%$ had diabetes and hypertension; $71.7 \%$ were not hospitalized in the last five years; 56.6 eat vegetables; $88.7 \%$ follow the recommended diet; $81.10 \%$ are non-smokers; $90.6 \%$ do not drink alcoholic beverages and $49.1 \%$ do not practice physical activity. Conclusion: the elderly have favorable health conditions.
\end{abstract}

Keywords: Elderly, Arterial hypertension, Diabetes mellitus, Health conditions.

\footnotetext{
${ }^{1}$ Graduanda do $10^{\circ}$ semestre de enfermagem pela Faculdade Independente do Nordeste - FAINOR. mary-lia-12@ hotmail.com;

${ }^{2}$ Doutora em Ciências da Saúde pela UFRN. Professora Titular do Programa de Pós - Graduação Stricto Sensu em Memória: Linguagem e Sociedade e do Curso de Fisioterapia da UESB. Professora da Faculdade Indenpendente do Nordeste - FAINOR. E-mail: lucianauesb@yahoo.com.br

${ }^{3}$ Doutoranda em Memória: Linguagem e Sociedade da Universidade Estadual do Sudoeste da Bahia - UESB. Docente do curso de Enfermagem da Faculdade Independente do Nordeste-FAINOR.polly_vl@yahoo.com.br
} 
Id on Line Revista Multidisciplinar e de Psicologia

Id on Line Multidisciplinary and Psycology Journal

\section{Introdução}

Conforme o Instituto Brasileiro de Geografia e Estatística (IBGE), o número de idosos no Brasil só vem aumentando, passando de 12,6\% em 2012 para 13\% em 2013, totalizando uma média de 26,1 milhões de idosos em todo o Brasil. Da mesma forma que tem ocorrido o aumento de idosos no Brasil, tem crescido o número de casos de Doenças Crônicas Não Transmissíveis (DCNT), especialmente nessa faixa etária (BRASIL, 2014).

De acordo os dados emitidos pelo Ministério da Saúde (MS) e pelo que Instituto Brasileiro de Geografia e Estatística (IBGE) 40\% da população adulta brasileira já manifestou algum tipo de DCNT, sendo as mulheres as mais atingidas (44\%), contra 33,4\% para os homens (BRASIL, 2014). Vale lembrar que são esses adultos, os futuros idosos no Brasil vivenciando essa realidade.

Entre as DCNT, a Hipertensão Arterial Sistêmica (HAS) e o Diabetes mellitus (DM), vem aumentando drasticamente em todo o mundo (WHO, 2012) e são as mais comuns no Brasil, constituindo a primeira causa de hospitalizações no sistema público de saúde. Além disso, são as duas patologias que mais acometem os idosos (BRASIL, 2013a). A HAS e o DM estão entre os cinco principais fatores de riscos para a mortalidade no mundo, no sentido que aumenta o risco de desenvolvimento de outras patologias crônicas (WHO, 2009).

Conforme a Pesquisa Nacional de Saúde de 2013, 44,4\% das pessoas de idade entre 60 e 64 anos, referiram diagnóstico de hipertensão, 52,7\% entre as pessoas de 65 a 74 anos de idade e de 55,0\% entre as pessoas de 75 anos ou mais de idade. No caso do DM não foi diferente, pois conforme a mesma pesquisa quanto maior a faixa etária, maior o percentual. Este variou de 0,6\%, para aqueles de 18 a 29 anos de idade, a 19,9\%, para as pessoas de 65 a 74 anos de idade. Para aqueles que tinham 75 anos ou mais de idade, o percentual foi de 19,6\% (IBGE, 2014).

Sabe-se que avaliação constante do estado de saúde de pessoas portadoras de HAS e DM, assim como o perfil social, demográfico e econômico contribuem para medidas de controle de possíveis complicações (ALMEIDA; GUTIERREZ; MARQUES, 2012). Nesse sentido, que a preocupação com as condições de saúde do idoso tem motivado o desenvolvimento de vários estudos sobre o envelhecimento humano. As condições de saúde são ocorrências na saúde das pessoas que se apresentam persistentemente e que requer respostas sociais reativas ou proativas, de maneira temporária ou contínua (MENDES, 2012). 
Diante do supracitado, percebe-se que a avaliação das condições de saúde é extremamente importante para controle e tratamento da HAS e do DM. Isto porque, as condições de Saúde é um excelente preditor para avaliar como está o processo de envelhecimento de uma população, especialmente quando se trata de uma população idosa portadora de duas doenças crônicas, tão comuns no Brasil, e que tem apresentado números alarmantes nos últimos anos.

Este estudo justifica-se pela necessidade de conhecer idosos portadores dessas doenças e como as condições de saúde destes podem influenciar na evolução ou melhora das mesmas. Trata-se de uma pesquisa relevante também para os profissionais de saúde que estão envolvidos nos cuidados para com os idosos, pois irá norteá-los quanto à medida que devem ser oferecidos aos mesmos, devido às limitações e sequelas desencadeadas pelas DCNT, foco deste estudo, podendo ainda ser utilizada como ferramenta de pesquisa avaliativa por compor dados informativos.

Diante do exposto, este estudo tem como objetivo investigar as condições de saúde de idosos portadores de Hipertensão Arterial Sistêmica e de Diabetes Mellitus de uma Estratégia de Saúde da Família de um município do interior da Bahia.

\section{Metodologia}

Trata-se de um estudo transversal do tipo descritivo, com abordagem quantitativa que foi realizado em uma Estratégia de Saúde da Família (ESF) de uma cidade do interior da Bahia.

Os participantes do estudo foram 53 idosos de 60 anos ou mais de ambos os sexos, hipertensos e/ou diabéticos, cadastrados no programa HIPERDIA da ESF já referida, que apresentaram condições mentais para responder aos questionários e que aceitaram participar do estudo.

Destaca-se que o Hiperdia destina-se ao cadastramento e acompanhamento de portadores de HAS e/ou DM atendidos na rede ambulatorial do Sistema Único de Saúde (SUS). É um programa que possibilita gerar informação para aquisição, dispensação e distribuição de medicamentos de forma regular e sistemática a todos os pacientes cadastrados (DATA SUS, 2016). 
Id on Line Revista Multidisciplinar e de Psicologia

Id on Line Multidisciplinary and Psycology Journal

Na ESF encontra-se cadastrados 3076 usuários, destes 90\% são idosos (pessoas a cima de 60 anos), totalizando 2.768,40. Sendo 475 portadores de HAS e 97 portadores de DM. Essa ESF atende usuários da zona urbana e rural. Portanto, para este estudo foram incluídos apenas idosos da zona urbana, devido especialmente a logística do estudo. A seleção da amostra foi realizada por meio de amostragem aleatória e considerado aqueles idosos que se apresentarem no dia da reunião do Hiperdia durante dois meses; que tinha cognição preservada (avaliada pelo MEEM) e que aceitaram participar do estudo voluntariamente.

A coleta de dados ocorreu na própria ESF, entre o mês de setembro e outubro, em uma sala reservada, nos dias das reuniões do Hiperdia e de forma complementar por meio de visitas domiciliares, quando necessário.

Para a execução dos objetivos propostos ao longo do trabalho, foram aplicados três instrumentos de pesquisa: o Mini Exame do Estado Mental (MEEM); um questionário Sócio demográfico e Econômico e um questionário de Condições de saúde.

Para avaliação do estado mental foi aplicado o MEEM, o qual avalia quatro parâmetros: memória a curto e longo prazo; orientação; informações do cotidiano; e capacidade de cálculo. Esse Mini-exame do estado mental foi utilizado apenas para a exclusão dos idosos que não apresentaram cognição para responder às perguntas da entrevista.

A fim de caracterizar o perfil sociodemográfico e econômico da população em estudo foi aplicado o questionário sociodemográfico e econômico. Os dados foram relacionados à: idade, sexo, cor da pele, estado civil, renda, moradia, escolaridade, religião e ocupação.

O questionário de Condições de Saúde contém 14 questões, referente a diagnóstico médico, histórico familiar para alguma patologia, se possui sequela de alguma doença, se já foi internado, hábitos alimentares, se tem alergia, se é tabagista e se toma medicações.

Posteriormente a coleta de dados foi realizada análise descritiva, por meio da distribuição de frequência absoluta e relativa para as variáveis categóricas. Foram fixados valores de confiança de $5 \%(\alpha=0,05)$ em todas as análises. Com o intuito de elaborar o banco de dados foi utilizado o aplicativo EPIDATA e, para a análise dos dados, o programa estatístico SPSS ${ }$, versão 20.0. Por meio das informações alcançadas foi realizada uma interpretação com vistas a alcançar o objetivo do estudo.

Todos os aspectos éticos e legais da Resolução 466/12 do Ministério da Saúde foram seguidos rigorosamente. Inicialmente o projeto foi encaminhado para o coordenador 
responsável da instituição onde ocorreu o estudo. Após a aprovação e assinatura da Autorização Institucional para a coleta de dados e Declaração de Infraestrutura o projeto foi submetido ao Comitê de Ética em Pesquisa da Faculdade Independente do Nordeste (FAINOR); via Plataforma Brasil, com parecer de aprovação $\mathrm{n}^{\circ}$ 1.687.752. Somente após a aprovação do Comitê de Ética foi dado início a coleta de dados. Todos os objetivos do estudo e os procedimentos da coleta foram explicados aos idosos. Aqueles que aceitaram participar do estudo assinaram um Termo de Consentimento Livre e Esclarecido (TCLE).

\section{Resultados}

Na Tabela 1 foram apresentados os dados referentes à caracterização sociodemográfica dos idosos. Observou-se predominância de idosos do sexo feminino $(53,7 \%)$, pardos $(64,8 \%)$, católicos $(74,0 \%)$, que sabem ler e escrever (51,9\%), com nenhuma escolaridade $(48,1 \%)$, casado/morando junto $(59,3 \%)$ e com média de idade de $71,68( \pm 13,96)$ anos.

\section{Tabela 1 - Caracterização sociodemográfica dos idosos}

\begin{tabular}{lcr}
\hline & N & $\%$ \\
\hline Sexo & & \\
Masculino & 25 & 46,3 \\
Feminino & 29 & 53,7 \\
& & \\
Cor & & \\
Parda & 35 & 64,8 \\
Branca & 14 & 25,8 \\
Negra & 2 & 3,8 \\
Amarela & 3 & 5,6 \\
& & \\
Religião & & \\
Católica & 40 & 74,0 \\
Protestante & 14 & 26,0 \\
& & \\
Sabe ler e escrever & & \\
Sim & 28 & 51,9 \\
Não & 26 & 48,1
\end{tabular}

Escolaridade

Nenhuma $26 \quad 48,1$

Ensino fundamental $\quad 23 \quad 42,6$

Ensino fundamental $2 \quad 3 \quad 5,6$

Ensino completo $\quad 2 \quad 3,7$ 
Id on Line Revista Multidisciplinar e de Psicologia

Id on Line Multidisciplinary and Psycology Journal

\begin{tabular}{lcr} 
Estado conjugal & & \\
Casado/morando junto & 32 & 59,3 \\
Viúvo & 16 & 29,6 \\
Divorciado/separado & 3 & 5,5 \\
Nunca casou & 3 & 5,6 \\
\hline Total & 54 & 100 \\
\hline
\end{tabular}

Fonte: Dados da pesquisa, 2016.

Na Tabela 2 é apresentada a distribuição dos idosos de acordo com profissão, renda pessoal e renda familiar. Verificou-se uma maior frequência de idosos com tipo de trabalho durante a vida referente a trabalho rural $(37,0 \%)$, origem de sustento da família relativo a aposentadoria (81,5\%), com renda mensal do idoso de 1 salário mínimo $(79,6 \%)$ e com renda mensal das pessoas da casa de 1 salário mínimo $(55,61 \%)$.

Tabela 2 - Distribuição dos idosos de acordo com profissão, renda pessoal e renda familiar.

\begin{tabular}{lcc}
\hline & N & $\%$ \\
\hline Tipo de trabalho durante a vida & & \\
Trabalho rural & 20 & 37,0 \\
Dona de casa & 12 & 22,2 \\
Funcionário Público & 8 & 14,8 \\
Outros & 14 & 26,0
\end{tabular}

Origem do sustento da família

Trabalho

$2 \quad 3,7$

Aposentadoria

$44 \quad 81,5$

Pensão

$4 \quad 7,4$

$\begin{array}{lll}\text { Ajuda de outros } & 2 & 3,7\end{array}$

$\begin{array}{lll}\text { Outros } & 2 & 3,7\end{array}$

\section{Renda mensal}

Nenhuma

$1 \quad 1,9$

1 salário mínimo

$43 \quad 79,6$

2 salários mínimos

$6 \quad 11,1$

Menos de 1 salário mínimo

$4 \quad 7,4$

\section{Renda mensal das pessoas da casa}

\begin{tabular}{lcr} 
1 salário mínimo & 30 & 55,6 \\
2 salários mínimos & 19 & 35,2 \\
3 salários mínimos & 2 & 3,7 \\
Sem renda & 3 & 5,5 \\
\hline Total & 54 & 100,0 \\
\hline
\end{tabular}

Fonte: Dados da pesquisa, 2016. 
Na Tabela 3, estão descritas a distribuição das condições de saúde dos idosos. Percebese uma maior distribuição de idosos com HAS $(66,0 \%)$, que fazem uso de medicamentos (100,0\%), sem sequela decorrente de doenças $(86,8 \%)$, com casos de doenças crônicas na família referente a HAS (45,3\%), que fez cirurgia (58,5\%), não internados nos últimos 5 anos $(71,7 \%)$ e que consume com frequência verduras $(56,6 \%)$.

Tabela 3 - Distribuição das condições de saúde dos idosos.

\begin{tabular}{lcr}
\hline & N & \% \\
\hline Doenças Crônicas & & \\
Hipertensão arterial sistêmica (HAS) & 35 & 66,0 \\
Diabetes Mellitus (DM) & 3 & 5,7 \\
HAS +DM & 12 & 22,6 \\
Câncer (CA) + HAS + DM & 2 & 3,8 \\
Outras & 1 & 1,9
\end{tabular}

\section{Uso de medicamentos}

Sim

53100,0

Sequela decorrente de doenças

Sim

Não

Histórico de doenças crônicas na família

HAS

DM

HAS +DM

$15 \quad 28,3$

$\mathrm{HAS}+\mathrm{DM}+\mathrm{CA}$

$5 \quad 9,4$

CA

$1 \quad 1,9$

$\mathrm{HAS}+\mathrm{CA}$

$6 \quad 11,3$

Fez cirurgia

Sim

Não

Internamento nos últimos 5 anos

Sim

$15 \quad 28,3$

Não

Consome com frequência

Frutas

$6 \quad 11,3$

Legumes

$3 \quad 5,7$

Verduras

$30 \quad 56,6$

Fritura

Massa

$\begin{array}{rr}9 & 17,0 \\ 53 & 100,0\end{array}$

Fonte: Dados de pesquisa, 2016. 
Na Tabela 3 foram demonstrados a distribuição dos idosos segundo hábitos de vida. Constou-se uma maior distribuição de idosos que seguem dieta recomendada por equipe de saúde $(88,7 \%)$, não fumante $(35,8 \%)$, que não consumem bebida alcoólica $(47,2 \%)$, não realizam atividade física $(49,1 \%)$, dorme bem $(60,4 \%)$, não possui alergia $(83,0 \%)$ e que acha sua saúde boa $(50,9 \%)$, segundo dados da Tabela 4 .

Tabela 4 - Distribuição dos idosos segundo hábitos de vida.

\begin{tabular}{|c|c|c|}
\hline & $\mathrm{N}$ & $\%$ \\
\hline \multicolumn{3}{|c|}{ Dieta recomendada por equipe de saúde } \\
\hline Sim & 47 & 88,7 \\
\hline Não & 2 & 3,8 \\
\hline Às vezes & 4 & 7,5 \\
\hline \multicolumn{3}{|l|}{ Fumante } \\
\hline Sim & 10 & 18,9 \\
\hline Não & 19 & 81,10 \\
\hline Já fumou & 24 & 45,3 \\
\hline Nunca fumou & 31 & 54,7 \\
\hline \multicolumn{3}{|c|}{ Consome bebida alcoólica } \\
\hline Sim & 5 & 9,4 \\
\hline Não & 50 & 90,6 \\
\hline Já bebeu & 23 & 43,4 \\
\hline Nunca bebeu & 32 & 56,6 \\
\hline \multicolumn{3}{|l|}{ Atividade física } \\
\hline $\operatorname{Sim}$ & 15 & 28,3 \\
\hline Não & 26 & 49,1 \\
\hline Às vezes & 12 & 22,6 \\
\hline \multicolumn{3}{|l|}{ Dome bem } \\
\hline Sim & 32 & 60,4 \\
\hline Não & 8 & 15,1 \\
\hline Às vezes & 12 & 22,6 \\
\hline Com medicação & 1 & 1,9 \\
\hline \multicolumn{3}{|l|}{ Possui alergia } \\
\hline Sim & 9 & 17,0 \\
\hline Não & 44 & 83,0 \\
\hline \multicolumn{3}{|c|}{ O que acha de sua saúde } \\
\hline Boa & 27 & 50,9 \\
\hline Ótima & 5 & 9,4 \\
\hline Regular & 17 & 32,1 \\
\hline Ruim & 4 & 7,5 \\
\hline Total & 53 & 100,0 \\
\hline
\end{tabular}

Fonte: Dados da pesquisa, 2016. 
Id on Line Revista Multidisciplinar e de Psicologia

Id on Line Multidisciplinary and Psycology Journal

\section{Discussão}

A faixa etária priorizada neste estudo é de pessoas idosas, ou seja, que tem 60 anos ou mais. Como já mencionado anteriormente, é uma população que só tem crescido no Brasil e que tem um perfil bem característico, especialmente no que se refere à feminização da velhice, pois o que tem demonstrado é que as mulheres têm vivido mais dos que os homens (IBGE, 2015).

Dados do IBGE (2015) evidenciam que persiste a diferença entre a expectativa de vida masculina e a feminina. Isso ocorre, porque a expectativa de vida feminina são maiores com 76,1 de idade, do que para os homens com 68,5 de idade, favorecendo a feminização da velhice. Segundo Berzins (2003), isso acontece devido vários fatores que contribuem para longevidade da população feminina, principalmente, sua postura em relação à saúde/doença e a relação com serviço/saúde, já que as mulheres buscam mais esses serviços.

No presente estudo, conforme apresentado nos resultados, e seguindo a tendência do Brasil, o sexo feminino também foi predominante com 53,7\%. Resultado parecido foi encontrado por Manicoff (2015), pois foi obtido um percentual de 63,6\% para os idosos do sexo feminino.

O crescimento da população idosa já pode ser considerado um fenômeno evidente em todos os continentes atingindo um percentual de $75,5 \%$. Vale salientar que são grandes as dificuldades enfrentadas por essa população, pois a maior parte reside em zona rural e apresentam alguns fatores que podem contribuir para uma maior vulnerabilidade dos idosos, como dificuldade a busca nos serviços de saúde, falta de transporte, baixa renda e um nível reduzido de escolaridade (HEITOR; RODRIGUES; TAVARES, 2013).

Esse estudo mostrou também que por morarem ou por já terem morado uma boa parte da vida na zona rural, teve uma porcentagem de $37,0 \%$ de idosos que trabalhou durante a maior parte da vida em trabalho rural, com passar do tempo e a com o chegar da idade esse trabalho não pode ser mais exercido, levando a uma aposentadoria, onde cerca de $81,5 \%$ dos idosos são aposentados, com renda de um salário mínimo, segundo dados da Tabela 2.

No que se refere às condições de saúde, o estudo demonstrou que a maioria dos idosos $(66,0 \%)$ é portador de HAS. A HAS tem sido considerada como um problema de alta magnitude, especialmente pelo fato de ser de grande prevalência e muitas vezes de difícil diagnóstico (CAVALARI et al., 2012). Além de ser fator de risco para o desenvolvimento de 
Id on Line Revista Multidisciplinar e de Psicologia

Id on Line Multidisciplinary and Psycology Journal

doenças cardiovasculares, acidente vascular cerebral, insuficiência renal, morte prematura e incapacidade (WHO, 2013).

O estudo de Leite et al. (2010) chegou a resultado similar ao presente estudo, pois dos 85 idosos que participaram da pesquisa, $65,88 \%$ são portadores de HAS. A predominância de HAS entre os idosos também foi encontrado no estudo realizado por Sousa et al. (2015). Essa realidade do número alto de hipertensos é um fato no Brasil, especialmente no que se refere aos idosos, pois a HAS é a patologia mais comum dessa faixa etária (BARRETO; CARREIRA; MARCON, 2015).

Outra patologia referida pelos idosos foi o DM. Dos 53 idosos, 3,80\% são portadores de DM apenas e 28,30\% portadores de DM e HAS juntas. Esse achado, alerta uma preocupação que o Ministério da Saúde tem tido nos últimos anos, pois a associação da HAS e do DM, é ainda mais perigosa para possíveis complicações, isto porque o uso de medicamento é maior e a alimentação requer muito mais cuidados, o que por vezes não ocorre da maneira adequada. Além disso, a principal causa de morbi-mortalidade da população brasileira, segundo o Ministério da Saúde são as doenças cardiovasculares, onde dois dos principais fatores de risco é justamente a HAS e o DM (BRASIL, 2014).

Todavia, a literatura apresenta o DM compensado como fator positivo na busca pela melhoria da qualidade de vida, isso porque um idoso com uma ou mais doenças crônicas pode ser considerado saudável, quando comparado com idoso com as mesmas doenças, porém sem controle, com sequelas decorrentes e incapacidades associadas (NASRI, 2005).

Em relação ao uso de medicamentos para o tratamento de patologias, verificou-se que $100,0 \%$ dos idosos fazem uso de medicamentos todos os dias, ficando claro que utilizam o tratamento medicamentoso. No caso da HAS este tipo de tratamento é indicado, principalmente, para pessoas com alto risco cardiovascular ou mesmo com níveis pressóricos no estágio 2 , ou seja, $\mathrm{PA} \geq 160 / 100 \mathrm{mmHg}$ (BRASIL, 2013). No caso do DM, o primeiro tratamento de escolha, tanto o DM do tipo 1, quanto do tipo 2, é o medicamentoso (WHO, 2016). Porém, se faz necessário ressaltar que associado a esse tratamento, sempre é indicado mudança de estilo de vida, sendo parte fundamental no controle da HAS e do DM (BRASIL, 2013; WHO, 2016).

No Brasil cerca de 35,9\% da população, segundo a Pesquisa Nacional de Saúde (IBGE, 2013), obtêm pelo menos um medicamento para hipertensão por meio do programa Farmácia Popular. Sendo que destes $91,1 \%$ receberam recomendações médicas para redução de ingestão 
Id on Line Revista Multidisciplinar e de Psicologia

Id on Line Multidisciplinary and Psycology Journal

de sal, 87,4\% para manterem as consultas de acompanhamento regular; 88,4\% para seguir uma alimentação saudável e $84,7 \%$ para a manutenção do peso adequado.

No presente estudo, observou ainda que $86,8 \%$ dos idosos não obteve sequela decorrente de doenças e 71,7\% não ficou internado nos últimos 5 anos. Estes dois achados são um resultado positivo, haja vista, demonstrar que o tratamento está sendo seguido adequadamente, especialmente aliado a mudança no hábito alimentar, pois $56,6 \%$ dos idosos relataram que consomem com frequência verduras e apenas 9,4\% consomem frituras.

Ressalta-se que a maioria desses idosos, participantes do estudo, residiram boa parte da vida em área rural, e trabalharam como lavrador até a aposentadoria, o que possibilita o acesso mais fácil a alimentos saudáveis como verduras e frutas e a dificuldade de acesso maior a alimentos industrializados. Destaca-se que esse dado vai de encontro com o que declara o Ministério da saúde por meio da Política Nacional de Alimentação e Nutrição (BRASIL, 2013).

No que tange ao histórico de doenças crônicas na família, 45,3\% dos entrevistados disseram que tem casos de hipertensos e $28,3 \%$ que tem casos de diabetes e hipertensão juntas na família. O estudo de Santos e Moreira (2012), realizado em uma região do Nordeste Brasileiro, evidenciou resultado semelhante, em que o histórico familiar de HAS foi de 29,8\% e $14,7 \%$ de HAS e DM.

Quanto às condições de saúde autorreferidas a maior predominância foi de estado de saúde bom (50,9\%). Acredita-se que o fato do idoso hipertenso e/ou diabético declarar estado de saúde bom, tenha relação com o controle das patologias e o acesso a medicamento e acompanhamento na unidade de saúde, o que é um ponto extremamente positivo, haja vista, essa realidade demonstrar um controle de duas doenças tão comuns entre adultos e idosos no Brasil (BRASIL, 2014). Além do mais, está associado ao fato desses idosos aprenderem a conviver com as doenças e mostrarem-se preparadas para enfrentá-las, desenvolvendo estratégias de enfrentamento (ALBORGHETTI; OLIVEIRA; SILVÉRIO, 2012).

O estudo de Borges et al., (2014) também chegou a essa conclusão, isso porque 47,81\% referiram possuir boa saúde. Ressalta-se que este estudo investigou idosos portadores e não portadores de doenças crônicas, porém a sua maioria $(77,38 \%$ ) apresentou algum tipo de doença crônica e destes 56,20\% era hipertenso, semelhante ao presente estudo.

Quanto aos hábitos de vida constatou-se uma maior distribuição de idosos que seguem dieta recomendada por equipe de saúde $(88,7 \%)$. Sabe-se que esse é um grande desafio para os 
Id on Line Revista Multidisciplinar e de Psicologia

Id on Line Multidisciplinary and Psycology Journal

profissionais de saúde, uma vez que a maioria dos indivíduos tem dificuldade para modificálas. Portanto, o fato desses idosos manterem uma dieta recomenda favorece o equilíbrio das doenças crônicas apresentadas por eles.

Verificou-se também que 90,6\% dos idosos não consomem bebida alcoólica e 43,4\% já consumiu. Esse achado demonstra que o fato do idoso ser diagnosticado com uma ou mais patologias tem possibilitado uma mudança nos padrões de uso da bebida alcoólica, o que é benéfico para o tratamento, uma vez que o álcool é fator de risco para a HAS, pois a redução do consumo de álcool diminui a pressão arterial (BRASIL, 2013).

Os resultados do II Levantamento Nacional sobre os Padrões de Consumo de Álcool na População Brasileira (LENAD, 2014), corrobora com os dados encontrados neste estudo, isso porque os números de idosos abstêmios de álcool tem sido significativo.

Quanto ao hábito de fumar, $81,10 \%$ dos idosos não fuma e 45,3\% já fumou. Confirmando com este resultado, Zaitune et al. (2012) evidenciam que 87,8\% dos idosos não tem o hábito de fumar, mas 47,6 relataram que já fumaram uma boa parte da vida. No Brasil, tem reduzido o número de idosos fumantes e o que se percebe é que quanto mais idoso a redução é ainda maior (ZAITUNE et al., 2012). Esse ocorrido é principalmente devido a uma mudança cultural e de orientação quanto aos riscos do fumo, bem como, devido ao diagnóstico de DCNT, como a hipertensão e o diabetes, as quais necessitam de mudança no padrão de vida.

No que diz respeito à prática de atividade física, $49,1 \%$ dos idosos não realizam nenhum tipo, ou seja, são pessoas sedentárias. Segundo Sousa et al. (2015), o predomínio de idosos que não praticam atividade física é alarmante. Em seu estudo 62,0\% dos idosos de um Município do Pará, cadastrados em uma ESF também não realizam atividade física. A atividade física tem sido relacionada a diversos benefícios e entre os principais está a melhora no padrão da atividade do coração e o controle do peso (WAN; WONG, 2014).

A população brasileira com maior acometimento de DM são aqueles na faixa etária a cima dos 60 anos, em especial os que passam dos 70 e o maior fator de risco tem sido justamente o excesso de peso (WHO, 2016), por isso, é de suma importância o incentivo pelos profissionais de saúde a prática de alguma atividade física, de acordo com a condição e a capacidade do indivíduo. 
Id on Line Revista Multidisciplinar e de Psicologia

Id on Line Multidisciplinary and Psycology Journal

\section{Considerações Finais}

Buscando a questão central, o estudou priorizou investigar as condições de saúde de idosos portadores de Hipertensão Arterial Sistêmica e de Diabetes Mellitus de uma Estratégia de saúde da Família de um município do interior da Bahia.

A análise dos resultados permitiu cumprir com o objetivo proposto e chegar a algumas conclusões. Em relação às condições de saúde verificou-se que os idosos têm realizado o controle das patologias e seguido as recomendações propostas pela a equipe de saúde, assim como, tem mantido um padrão de redução dos fatores de risco, especialmente no que se refere ao consumo de bebidas alcoólicas e de fumo.

Além disso, foi demonstrado que os idosos têm mantido uma alimentação adequada, conforme a necessidade para as duas patologias, com redução de sal e de carboidratos e aumento de consumo de verduras, frutas e legumes.

Outro achado a salientar é que os idosos apresentam o conhecimento sobre as suas limitações por conta da patologia de base, pois tem seguido o uso de medicamentos adequadamente e de mudanças nos hábitos de vida. Todavia, se faz necessário salientar que poucos foram os idosos que relataram praticar atividade física, possibilitando, dessa forma, inferir que é muito importante que os profissionais da ESF, com apoio dos profissionais do Núcleo de Apoio a Saúde da Família (NASF), realizem ações estratégicas para mudar essa realidade e permitir ainda mais o controle das patologias.

Portanto, sugerem que sejam adotadas pela comunidade, principalmente pelas ESF, medidas capazes de averiguar e incentivar os idosos a manter os cuidados necessários para uma condição e hábitos de vida saudável. Tal conduta irá proporcionar melhores condições de saúde para os idosos, prolongando ainda mais a longevidade com qualidade de vida.

\section{Referências}

ALBORGHETTI, K. T.; OLIVEIRA, M. A. da S.; SILVÉRIO, M. R. Diabetes mellitus tipo II em pessoas idosas: estratégias utilizadas pelos usuários da rede pública de saúde em um município do Sul de Santa Catarina. RBCEH, Passo Fundo, v. 9, n. 2, p. 200-212, maio/ago. 2012. Disponível em: <http://seer.upf.br/index.php/rbceh/article/viewFile/200-212/pdf>. Acesso em: 04 abr. 2016. 
Id on Line Revista Multidisciplinar e de Psicologia

Id on Line Multidisciplinary and Psycology Journal

ALMEIDA, M, A, B; GUTIERREZ, G, L; MARQUES, R. Qualidade de vida: definição, conceitos e interfaces com outras áreas, de pesquisa. São Paulo: Escola de Artes, Ciências e Humanidades - EACH/USP, 2012. Disponível em: <http://each.uspnet.usp.br/edicoeseach/qualidade_vida.pdf>. Acesso em: 04 abr. 2016.

BARRETO, M. da S.; CARREIRA, L.; MARCON, S. S. Envelhecimento populacional e doenças crônicas: Reflexões sobre os desafios para o Sistema de Saúde Pública. Revista Kairós Gerontologia, São Paulo, SP, Brasil: FACHS/NEPE/PEPGG/PUC-SP, v. 18, n. 1, p. 325-339, jan./mar. 2015. ISSN 1516-2567. ISSNe 2176-901X.

BERZINS, M. A. V. S. Envelhecimento populacional: uma conquista para ser celebrada. Revista Serviço Social e Sociedade, n. 075, 2003.

BORGES, A. M.; SANTOS, G.; KUMMER, J. A.; FIOR, L.; MOLIN, V. D.; WIBELINGER, L. M. Autopercepção de saúde em idosos residentes em um município do interior do Rio Grande do Sul. Rev. Bras. Geriatr. Gerontol., Rio de Janeiro, v. 17, n. 1, p. 7986, mar. 2014. Disponível em: <http://www.scielo.br/scielo.php?script=sci_arttext\&pid=S1809-98232014000100079>. Acesso em: 04 abr. 2016.

BRASIL. Ministério da Saúde (MS). Secretaria de Atenção à Saúde. Departamento de Atenção Básica. Política Nacional de Alimentação e Nutrição. Brasília: MS; 2013.

BRASIL. Ministério da Saúde. Secretaria de Atenção à Saúde. Departamento de Atenção Básica. Estratégias para o cuidado da pessoa com doença crônica: hipertensão arterial sistêmica. Brasília: Ministério da Saúde, 2013. 128 p. (Cadernos de Atenção Básica, n. 37).

BRASIL. Ministério da Saúde. Secretaria de Atenção à Saúde. Departamento de Atenção Básica. Estratégias para o cuidado da pessoa com doença crônica: diabetes mellitus. Brasília: Ministério da Saúde, 2014. 160p. Disponível em: <http://bvsms.saude.gov.br/bvs/publicacoes/estrategias_cuidado_pessoa_diabetes_mellitu s_cab36.pdf>. Acesso em: 04 abr. 2016.

CAVALARI, E. et al. Adesão ao tratamento: estudo entre portadores de hipertensão arterial em seguimento ambulatorial. Revista Enfermagem UERJ. v. 20, n. 1, p. 67-72. 2012.

DATA SUS. HIPERDIA - Sistema de Cadastramento e Acompanhamento de Hipertensos e Diabéticos. Disponível e: http://datasus.saude.gov.br/sistemas-eaplicativos/epidemiologicos/hiperdia

HEITOR, S. F. D.; RODRIGUES, L. R.; TAVARES, D. M. dos S. Prevalência da adequação à alimentação saudável de idosos residentes em zona rural. Texto contexto enferm., Florianópolis, v. 22, n. 1 Jan./Mar. 2013. Doi: http://dx.doi.org/10.1590/S010407072013000100010

INSTITUTO BRASILEIRO DE GEOGRAFIA E ESTATÍSTICA (IBGE). Pesquisa Nacional de Saúde 2013: Percepção do estado de saúde, estilo de vida e doenças crônicas. Brasil, Grandes Regiões e Unidades da Federação. Ministério do Planejamento, Orçamento e Gestão. 
Id on Line Revista Multidisciplinar e de Psicologia

Id on Line Multidisciplinary and Psycology Journal

Diretoria de pesquisas. Coordenação de Trabalho e rendimento. Rio de Janeiro: 2014.

Disponível em: <ftp://ftp.ibge.gov.br/PNS/2013/pns2013.pdf>. Acesso em: 13 abr. 2016.

INSTITUTO BRASILEIRO DE GEOGRAFIA E ESTATÍSTICA (IBGE). Pesquisa

População estimada de tremedal para o ano de 2015. 2015. Disponível em:

<http://www.ibge.gov.br/home/estatistica/populacao/estimativa2015/estimativa_tcu.shtm>.

Acesso em: 12 abr. 2016.

LEITE, M. T. et al. Caracterização e condições de saúde de idosos mais idosos residentes em um município do norte do Rio Grande do Sul. RBCEH, Passo Fundo, v. 7, supl. 1, p. 71-79, 2010. Disponível em: <http://seer.upf.br/index.php/rbceh/article/view/1098/pdf>. Acesso em: 18 nov. 2016.

LENAD. II Levantamento Nacional de Álcool e Drogas - 2012. Supervisão Ronaldo Laranjeira et al. São Paulo: Instituto Nacional de Ciência e Tecnologia para Políticas Públicas de Álcool e Outras Drogas (INPAD), UNIFESP, 2014. Disponível em: <http://inpad.org.br/wp-content/uploads/2014/03/Lenad-II-Relat\%C3\%B3rio.pdf>. Acesso em: 18 nov. 2016.

MANICOFF, R. C. L. Estado nutricional de idosos participantes do sistema Hiperdia: características sociodemográficas e níveis pressóricos. Rev Rene, v. 16, n. 5, p. 746-53, set./out. 2015. Disponível em:

<http://www.periodicos.ufc.br/index.php/rene/article/viewFile/2845/2210>. Acesso em: 15 nov. 2016.

MENDES, E. V. O cuidado nas condições crônicas na atenção primária à saúde: o imperativo da consolidação da estratégia de saúde da família. Organização PanAmericana de Saúde, Brasília DF: 2012.NASRI JR., F. S. Diabtes Mellitus. In: RAMOS, L. R.; TONIOLO NETO, J. (Orgs.). Geriatria e gerontologia. Barueri, SP: Manole, 2005. p. 77 94.

SANTOS, J. C. dos; MOREIRA, T. M. M. Fatores de risco e complicações em hipertensos/diabéticos de uma regional sanitária do nordeste brasileiro. Rev Esc Enferm., USP; v. 46, n. 5, p. 1125-1132, 2012. Disponível em:

<http://www.scielo.br/pdf/reeusp/v46n5/13.pdf>. Acesso em: 11 abr. 2016.

SOUSA, F. de J. de; OLIVEIRA, M. de F. de; RAMOS, E. M. L.; GONÇALVES, L. H. T. Condições de vida e saúde de usuários idosos do programa d e saúde da família. Estud. Interdiscipl. Envelhec., Porto Alegre, v. 20, n. 1, p. 219-234, 2015.

WAN, M.; WONG, R. Y. Benefits of exercise in the elderly. CGS Journal of CME, v. 4, issue 1, 2014. Disponível em:

$<$ http://www.canadiangeriatrics.ca/default/index.cfm/journals/canadian-geriatrics-societyjournal-of-cme/cme-journal-vol-4-issue-1-2014/benefits-of-exercise-in-the-elderly/>. Acesso em: 11 abr. 2016. 
WORLD HEALTH ORGANIZATION (WHO). Global Health Risks: Mortality and burden of disease attributable to selected major risks. Geneva: WHO, 2009. 70 p. Disponível em: <http://www.ufrgs.br/psiquiatria/psiq/WHOQOL-OLD.pdf>. Acesso em: 11 abr. 2016.

WORLD HEALTH ORGANIZATION (WHO). Knowledge Translation on Ageing and Health: A framework for policy development, 2012. Disponível em: $<$ http://www.who.int/ageing/publications/knowledge_translation_en.pdf >. Acesso em: 03 abr. 2016.

World Health Organization. Health topics: Chronic diseases. Geneva: World Health Organization; 2013.

WORLD HEALTH ORGANIZATION (WHO). Informe Mundial sobre la Diabetes. Resumen de Orientación. World Report on Diabetes. Executive summary, 2016. Disponível em: http://www.who.int/diabetes/global-report/en/.

ZAITUNE, M. P. A. et al. Fatores associados ao tabagismo em idosos: inquérito de saúde no Estado de São Paulo. Cad. Saúde Pública, v. 28, n. 3, p. 583-595, mar. 2012. Disponível em: <http://www.scielosp.org/pdf/csp/v28n3/18.pdf>. Acesso em: 03 abr. 2016.

\section{Como citar este artigo (Formato ABNT):}

FERRAZ, M.O.; REIS, L.A.; LIMA, P.V. Condições de saúde de idosos portadores de Diabetes mellitus e hipertensão arterial sistêmica. Id on Line Revista Multidisciplinar e de Psicologia, Janeiro de 2017, vol.10, n.33, p. 56-71. ISSN: 1981-1179.

Recebido: 23/11/2016

Aceito: 24/11/2016 